\title{
Electrode modification techniques for organic electronics
}

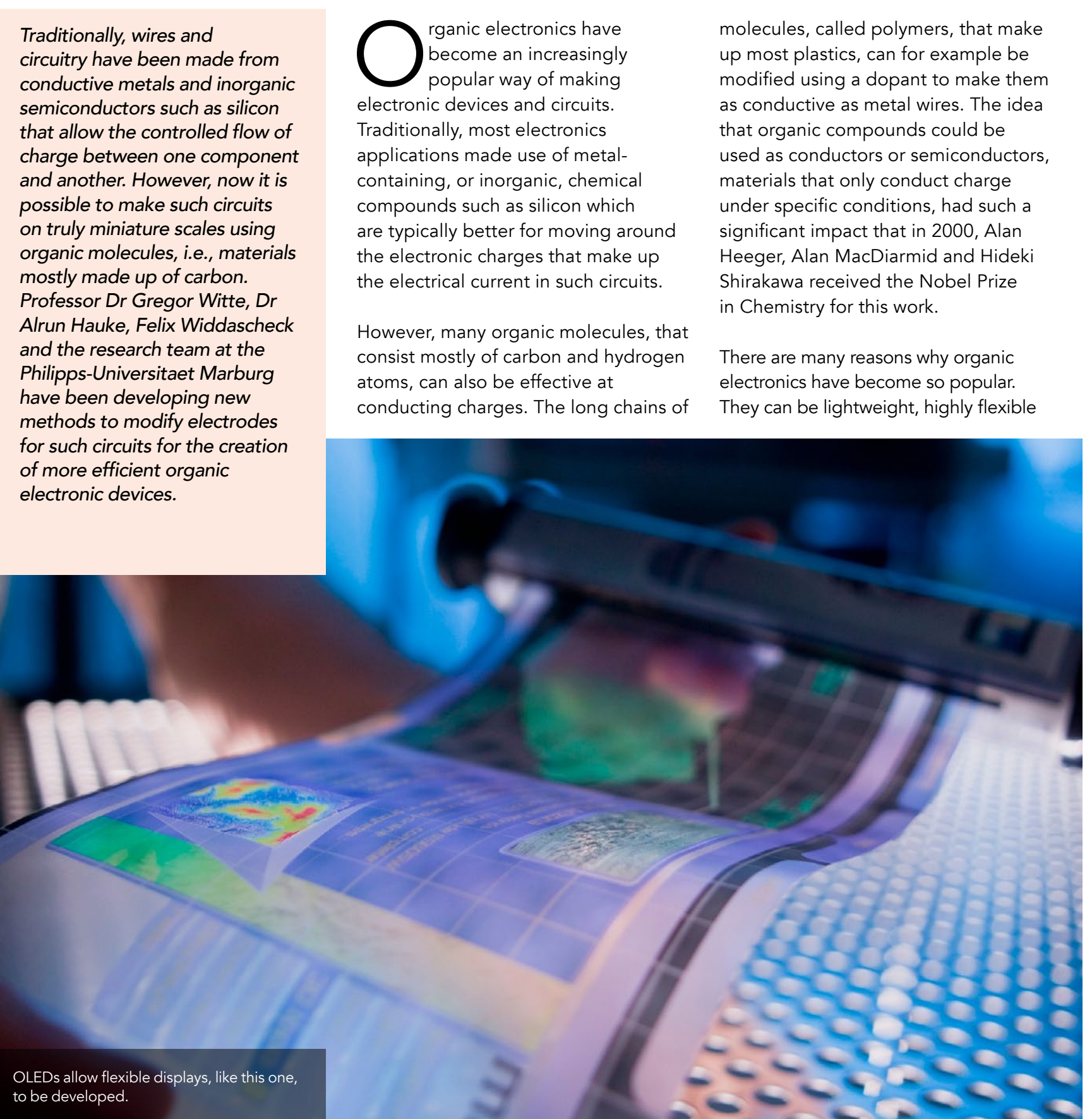

Traditionally, wires and for such circuits for the creation of more efficient organic to be developed.

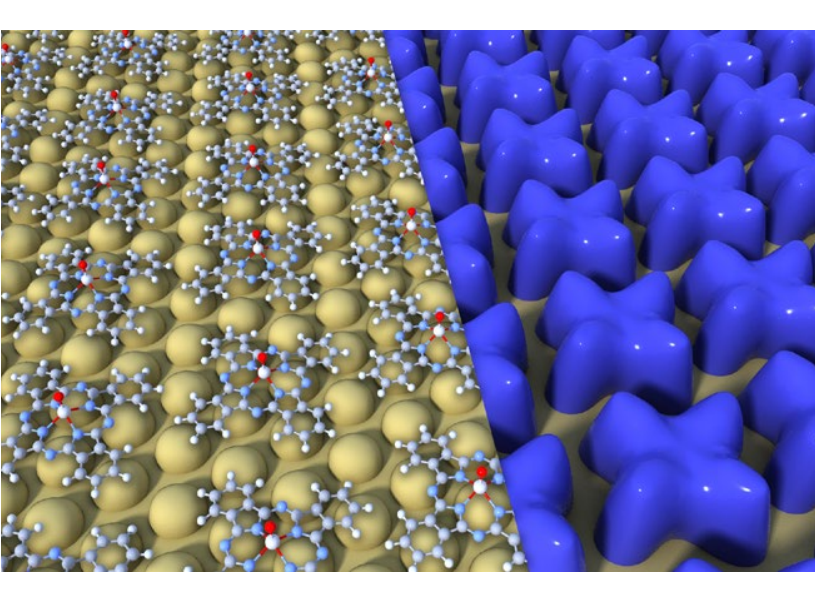

A layer of TiOPc molecules attaching to a single crystalline gold monolayer
(left) and forming a correspondingly well-ordered monolayer (right).

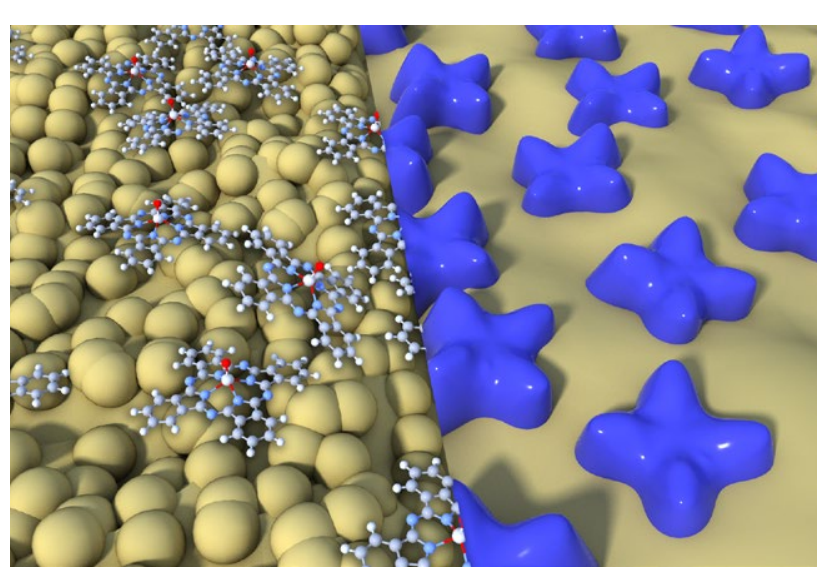

When the surface is rough, like a polycrystalline structure (left), the
corresponding monolayer of TiOP c is similarly disordered. and cheap to manufacture. Organic ight emitting diodes (OLEDs) are one of the major success stories of organic electronics - OLEDs are now found in most of the displays we use and have

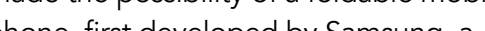
reality Furthermore, they can be used to make electronic paper, as the company Plastic Logic (Dresden, Germany) have shown using transistors (the type of small control switches which most modern technology is based on) made of organic semiconductors. And with steadily rising efficiencies, flexible organic solar cells integrated into bags or clothing will soon become a reality too.

The three colleagues at PhilippsUniversitaet Marburg are experts at finding new ways to improve how we make organic electronic devices. In particular, they develop novel strateg to improve the structural properties interfaces They are using different charas high-resolution microscopes, capable of imaging single molecules, X-ray diffraction and spectroscopy to really ty and understand in detail the complex structures of the organic semiconductor materials and then use this information to improve their design.

INTERFACING ISSUES One area that the team has bee focusing on is modffication of the metal electrodes. One of the key issues in organic electronics lies at the interface between conventional metal electrodes and the novel organic semiconducto the charge injector for the organic

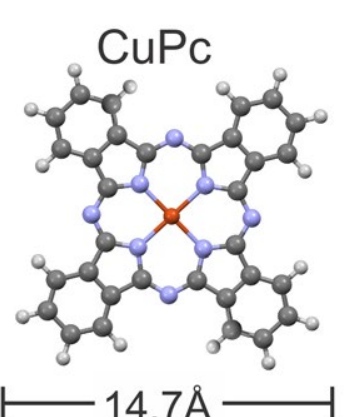

TiOPC

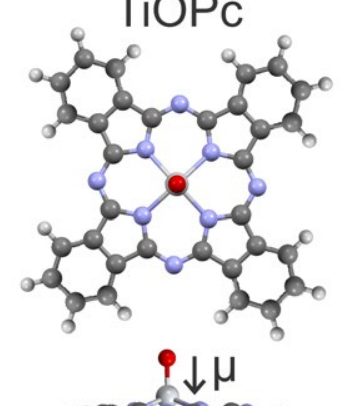

$\mathrm{F}_{16} \mathrm{CuPc}$ ${ }_{10}{ }_{10} \mathrm{CPC}_{2}$

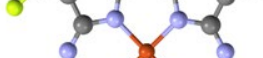
करे करेक $\longmapsto 14.7 \AA-$

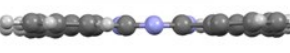
coserobrocos

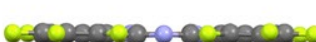

Organic light emitting diodes (OLEDs) are one of the major success stories of organic electronics.

semiconductor and the efficiency of (n) for the overall efficiency of the device. a measure for how easily charges can The interfal between the electrode and the semiconductor therefore is a crucial area to target for improving such devices. A useful measure in this context is the so-called work function difference between the metal and the organic semiconductor. Each has a be transferred from one material to the other. The team at Marburg have recently shown that they can change he work function of metal electrodes in a controlled fashion by adding a monolayer, on top of the metal.

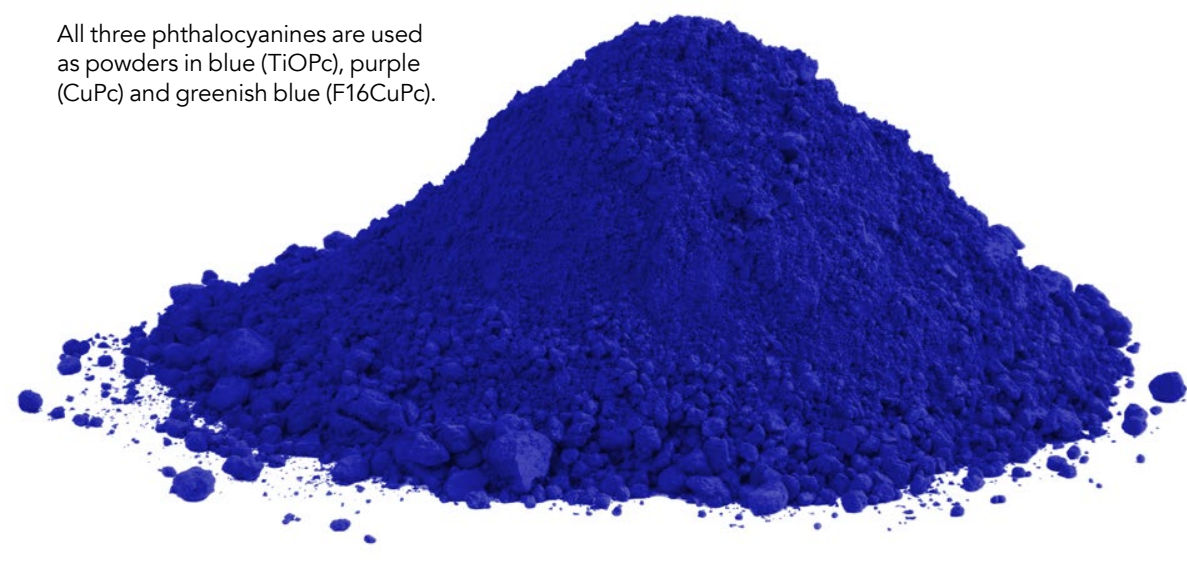




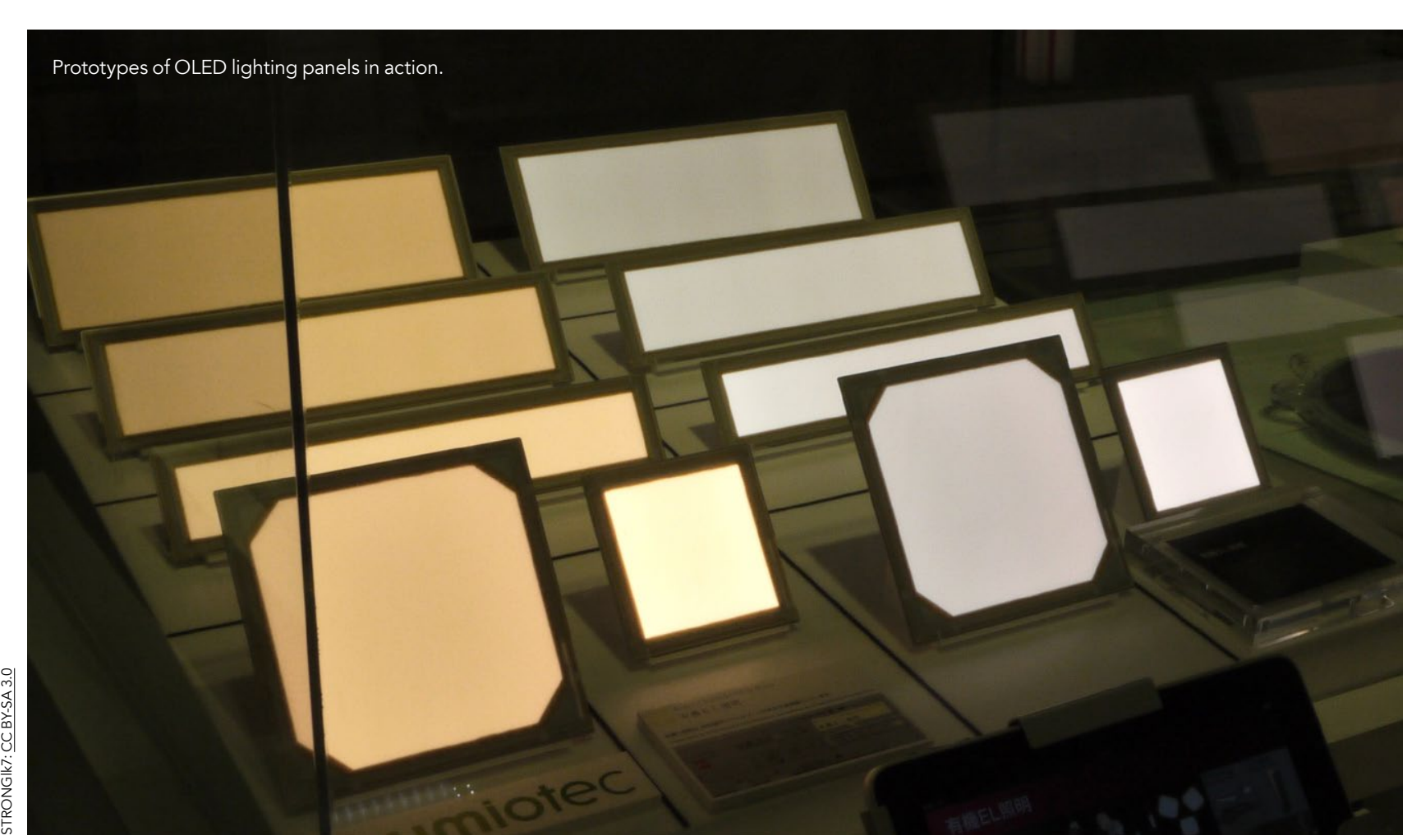

Control over the metal work function is important as this needs to be tailored for each organic semiconductor to make the most efficient devices. However, creation of these single organic monolayers is not easy. Usually these monolayers are applied in what is known as wet chemical process which involves reactive solvents and is therefore difficult to incorporate into the fabrication of high-performance devices. The team at Marburg uses organic molecular beam deposition in ultra-high vacuum instead, which suitable for the manufacture of

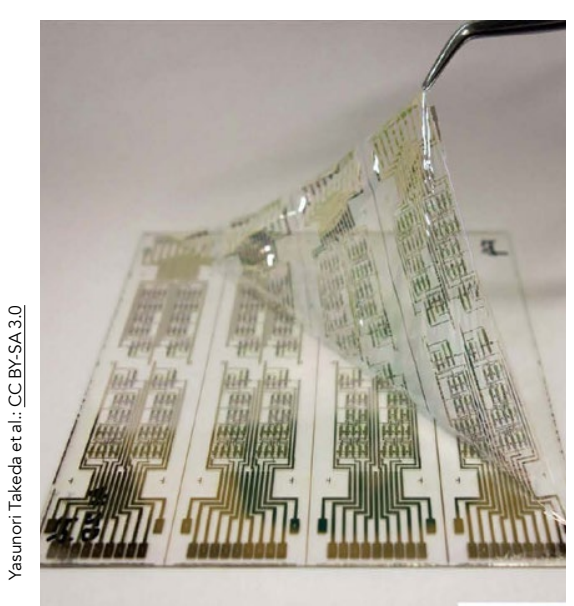

The total thickness of this organic circuiti is less

complex devices. This method, processes, has been used in the field needed and there is excellent control for years, also to make work-function Widdascheck have shown this approac

The Marburg team's method uses organic molecular beam deposition, which is more reliable, reproducible and suitable for the manufacture of complex devices.

modifying monolayers. The team goes is highly successful for depositing layers one step further in ensuring a precise of phthalocyanines, large molecules correlates the mocular arrangement onto metals such as siver, gold and copper. They have studied this both

observed work function changes.

ORGANIC MOLECULAR

BEAM DEPOSITION

Organic molecular beam deposition to very high temperatures inside a vacuum chamber so that they

form a vapour (this process is called

sublimation). When brought into

contact with the cold surface of the

metal electrode, molecules adsorb

and form a film on top of the meta.

What the Marburg team then does

is to heat the metal in order to remove

just the single desirs layers, until

\section{Behind the Research}

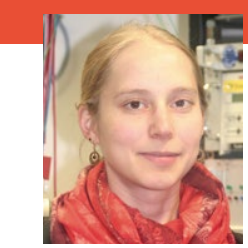

Dr Alrun Aline Hauke

E: alrun.hauke@physick

. terir structure can be manipulated to control optoelectronic properties.

\section{Detail}

Philipps-Universitaet Marburg

Fachbereich Phys

Renthof 7

Germany

Group website: https://www.uni-

marburg.de/en/fb13/molkk

Dr Alrun Aline Hauke obtained her Dresden in 2016 under her maiden

name Guenther and has since then been a PostDoc in the Molecular Solids group at Philipps-Universita Marburg. Her research interests include organic transistor architectur and their functional interfaces.

\section{间 \\ Felix \\ Widdascheck}

E: felix.widdascheck@physik.uni-marburg.de
Dr Gregor Witte regor.witte@physik.uni-marburg.de (n) it is highly adaptable for different

organic semiconductor. Choosing

different molecules for the monolayers

or tuning the layer thickness allows

to precisely enforce a specific work

function value in the metal electrodes

which is then compatible with a

or a specific organic device type.
Felix Widdascheck obtained his MSc at Philipps-Universitaet Marburg in 2018 in the Molecular Solids group student focusing on work function studies at metal-organic interfaces and the microscopic structure of

Prof Dr Gregor Witte has been a full university professor at Philippsis head of the Molecular Solids group at the department of physics. He studied physics at the Universitaet of Goettingen and received his PhD in at the Max-Planck-Institute of stays at IBM Almaden Research Cent
San Jose (USA) and Lawrence Berkeley National Laboratory, Berkeley (USA) Chemistry at the Universitaet Bochum where he was appointed a lecture after his habilitation. His research interests include the template and interface-controlled growth and optoelectronic properties of organic semiconductor films and the physical

\section{References}

Widdascheck, F., Hauke, A. A., \& Witte, G. (2019). A SolventFree Solution: Vacuum-Deposited Organic Monolayers Functional Materials, 29(17), 1-10. https:///doi.org/10.1002/ Functional Materias
adfm. 201808385

Mänz, A., Hauke, A. A., \& Witte, G. (2018). Copper Phthalocyanine as Contact Layers for Pentacene Films Grown 2165-2172 htps:/doi no 101021/2s.jpc 7b10324

\section{Personal Response}

ts organic beam deposition scalable to mass produce such devices?

II We certainly think so and so do the people at the company Heliatek (Dresden, Germany) who are one of the world's leading R\&D companies manufacturing efficient, large-area organic solar cell prototypes. The question you high throughput and low quality or whether you are after high-performance devices for sophisticated applications. In the latter case, OMBD is most likely your best option, since the cheaper solvent-based methods are less

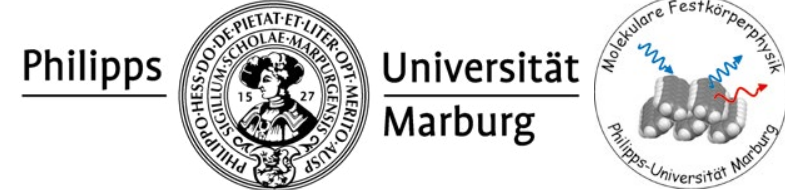

